\title{
Prognostic Factors in Non-Hodgkin's Lymphoma Patients Treated by Autologous Stem Cell Transplantation: A Single Center Experience
}

\author{
Cheolwon Suh, M.D. ${ }^{1}$, Sang Hee Kim, M.D. ${ }^{1}$, Hyo Jung Kim, M.D. ${ }^{3}$, Geundoo Jang, M.D. ${ }^{1}$, Eun Kyung \\ Kim, M.D. ${ }^{1}$, Ok Bae Ko, M.D. ${ }^{1}$, Shin Kim, R.N. ${ }^{1}$, Hee Jung Sohn, M.D. ${ }^{1}$, Jung Shin Lee, M.D. ${ }^{1}$, M. Wookun \\ Kim, M.D. ${ }^{1}$ and Jooryung Huh, M.D. ${ }^{2}$ \\ Departments of ${ }^{1}$ Internal Medicine and ${ }^{2}$ Pathology, Asan Medical Center, University of Ulsan College of Medicine, Seoul, \\ ${ }^{3}$ Department of Internal Medicine, College of Medicine, Hallym University Sacred Heart Hospital, Anyang, Korea
}

Purpose: Autologous stem cell transplantation (ASCT) is increasingly used in patients with non-Hodgkin's lymphoma (NHL). Various clinical parameters-were evaluated to obtain significant predictors of the outcome following ASCT in patients with NHL.

Materials and Methods: Between April 1994 and December 2003, ASCT was performed on 80 patients with NHL at the Asan Medical Center.

Results: Patients had various histological subtypes and disease status. The two year progression free survival (PFS) and overall survival for all patients were 34 and $31 \%$, respectively. A univariate analysis showed the performance status, stage, modified extranodal involvement category, International Prognostic Index (IPI) at mobilization, disease status at mobilization, and history of radiation prior to mobilization as significant predictors of the outcome following ASCT. Four risk

\section{INTRODUCTION}

The potential for cure of a non-Hodgkin's lymphoma (NHL) in adults is probably less than $30 \%$ (1). In 1978, NHL was the first disease where high dose therapy, supported with the re infusion of autologous stem cells was used (2), indicating the ability of dose intensification for the cure of some patients with a relapsing or refractory disease (3). NHL is currently the second most frequent indication for autologous stem cell transplantation (ASCT). ASCT provides an increasing number of NHL patients the best opportunity for cure under certain circumstances. However, many patients still relapse after ASCT, despite the therapeutic advances over the last two decades.

Correspondence: Cheolwon Suh, Department of Internal Medicine, Asan Medical Center, 388-1 Pungnap2-dong, Songpa-gu, Seoul 138-736, Korea. (Tel) 82-2-3010-3209, (Fax) 82-2-3010-6961, (Email) csuh@amc.seoul.kr

Received April 18, 2005, Accepted August 11, 2005 groups, with different 2 year PFS, were identified by the age adjusted IPI at mobilization (mAAIPI): low risk 44\%; low intermediate risk $40 \%$; high intermediate risk $19 \%$; and high risk $0 \% \quad(p=.0003)$. A multivariate analysis revealed 3 significant factors for the PFS: disease status, prior RT and MAAIPI.

Conclusion: The mAAIPI was found to be an independent predictor of the outcome of NHL patients undergoing ASCT. This powerful prognostic tool should be used to evaluate potential candidates for ASCT. (Cancer Res Treat. 2005;37:294-301)

Key Words: Non-Hodgkin's lymphoma, Autologous peripheral blood stem cell transplantation, Prognosis, Hematopoietic stem cell mobilization

Many ways have been explored to improve the outcome of ASCT in NHL patients, with new approaches continually being investigated.

The data of ASCT should be interpreted with caution, as the patient populations studied are a very exclusive group. In addition, due to differences in the selection criteria, transplant regimens and follow up, the comparison of studies is sometimes difficult (1). Numerous factors have been identified as significant in predicting patients' responses to transplantation, with the most useful of these being the sensitivity of the lymphoma to chemotherapy (3). The most important factors among the other variables associated with a poor outcome include elevated lactate dehydrogenase (LDH) (4), extensive previous treatment $(5,6)$, poor performance status (7) and high grade histology (5).

The International Prognostic Index (IPI) is a well established scoring system for predicting the survival in aggressive NHL (8). When the IPI was applied to all newly diagnosed patients over a 9 year period, it was applicable to unselected NHL patients for any grade of the Working Formulation (9). The IPI has been evaluated by many investigators in NHL patients treated with high dose therapy and ASCT $(10 \sim 13)$. These studies assessed the value of the IPI in patients with selected 
histological subtypes, with each study evaluating its own specific disease status or clinical situations. Clinical trials evaluating the clinical efficacy of ASCT might include any type of NHL and disease status. Here, the analysis of 80 patients with NHL treated by ASCT is reported, and shows that the age adjusted IPI at mobilization (mAAIPI) was an independent predictor of the outcome following ASCT.

\section{MATERIALS AND METHODS}

\section{1) Patients}

Between April 1994 and December 2003, 80 patients eligible for ASCT were enrolled at the Asan Medical Center using Institutional Review Board approved protocols for NHL. The written informed consent was obtained from each patient. All histological specimens were reviewed by a pathologist. Before 1998, the histopathology of specimens was classified according to the International Working Formulation $(14,15)$, but subsequent diagnosis have been performed using the World Health Organization/Revised European American Lymphoma (WHO/ REAL) classification; therefore, those diagnosed before 1998 were classified retrospectively according to the WHO/REAL system (15).

All patients were staged according to the Ann Arbor system, and the response assessed based on the recommendations from the NCI sponsored international working group (16).

\section{2) Eligibility criteria for ASCT}

Patient eligibility criteria for ASCT included the following: 1) biopsy proven NHL; 2) aged 15 to 65 years; 3) adequate cardiac function, as defined by an ejection fraction greater than or equal to $45 \%$; pulmonary function, as defined by a diffusion capacity greater than or equal to $50 \%$ of that predicted; renal function, as defined by a serum creatinine level less than or equal to $2 \mathrm{mg} / \mathrm{dl}$; hepatic function, defined as an AST/ALT ratio and alkaline phosphatase of less than twice the upper normal limit and a serum bilirubin level of less than or equal to $2 \mathrm{mg} / \mathrm{dl}$; 4) negative serology for human immunodeficiency virus; no history of other malignancies; and no NHL involvement of the central nervous system. At the time of peripheral blood progenitor cell (PBPC) mobilization, the disease status of the patients was re evaluated, and classified as either a complete response, partial response, chemotherapy sensitive relapse or refractory (refractory relapse and primary refractoriness). After 2 cycles of conventional salvage chemotherapy, patients with complete or partial responses were defined as having chemotherapy sensitive relapses; all other responses were classified as refractory relapses. Patients with high intermediate or high IPI scores on initial diagnosis were offered ASCT once a complete response had been attained.

\section{3) PBPC mobilization}

When a patient was assessed as a suitable candidate for ASCT, PBPC mobilization and collection was performed, as previously described (17).

\section{4) IPI at PBPC mobilization (mIPI) and mAAIPI}

The mIPI, assessed prior to the initiation of PBPC mobilization, was comprised of 5 risk factors: age older than 60 years,
LDH more than the normal upper limit, an ECOG performance status greater than 1 , a stage III or IV disease and more than 1 extranodal site. For patients showing a complete response, the stage was assigned as zero, and scored as " 0 " in the IPI system. The mAAIPI, assessed prior to the mobilization of PBPC, comprised of 3 elements of the IPI risk factors: LDH, stage and the ECOG performance status. These were identical to the predictive model for an aggressive non Hodgkin's lymphoma of the International Non Hodgkin's Lymphoma Prognostic Factors Project (8). The mAAIPI groups were assigned to low risk if they had no factor, low intermediate risk with 1 factor, high intermediate risk with 2 factors, and high risk if all 3 factors were present.

\section{5) ASCT procedure}

The BEAM or BEAC regimen was used as a high dose conditioning regimen for NHL $(18,19)$. Lenograstim was used to facilitate engraftment (19). Patients were cared for in a single room, with the strict maintenance of reverse isolation for the prevention of infectious complications. All patients were administered prophylactic antimicrobials, which consisted of ciprofloxacin, fluconazole and acyclovir. Patients received transfusions of red blood cells and platelets where clinically indicated.

\section{6) Follow up}

Assessment of response to ASCT was performed by repeating the examinations of previously involved sites. Subsequent follow up procedures included physical examinations every 3 months for the first 2 years, then every 6 months for 3 years, and annually thereafter. Thoracic and abdominal CT scans were performed every 6 months during the first 2 years and annually thereafter for 3 years.

\section{7) Statistical analysis}

The primary endpoint was progression free survival (PFS), as calculated from the ASCT to the date of disease progression, relapse from a complete response, death, or to the date of last contact. Overall survival (OS), the time from ASCT until last follow up or death, was also evaluated. Survival analyses were performed using the Kaplan and Meier method. The log rank test was used to compare the survival distributions. Potential predictive factors of survival were entered into a multivariate analysis using the Cox proportional hazards model. All statistical analysis was performed using SPSS version 10.1 (SPSS, Chicago, IL).

\section{RESULTS}

\section{1) Patient characteristics}

A total of 80 patients with NHL were eligible for the analyses. The demographic features of the patients are listed in Table 1. The median age at PBPC mobilization was 39 years, ranging from 16 to 65 years, with the study group comprised of 29 women and 51 men. Forty-five and 35 patients had B-cell and T-cell NHL, respectively. Of those with B-cell NHL, the histologies included 32 diffuse large cell lymphomas, 7 Burkitt lymphomas, 3 mantle cell lymphomas, 2 follicular lymphomas and 1 nodal marginal zone lymphoma; those with T-cell NHL 
A

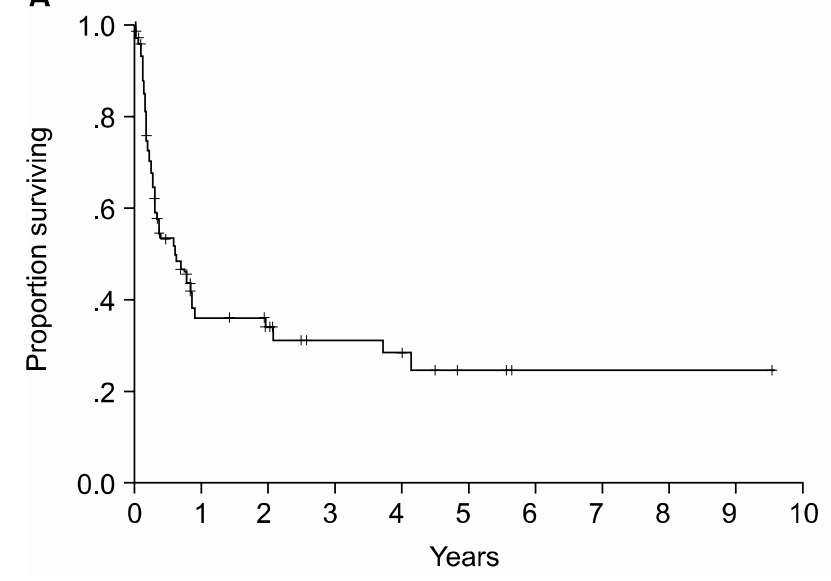

B

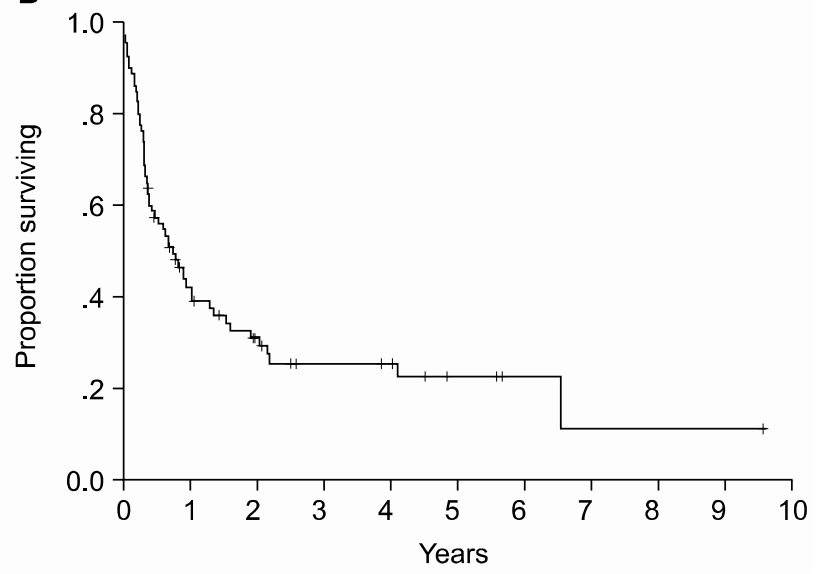

Fig. 1. Progression free survival (A) and overall survival (B) for all 80 patients. The Kaplan Meier estimate of the proportion of patients remaining progression free at median follow up of 2 years following ASCT was 34\%. And overall survival at 2 years was estimated as $31 \%$.

comprised of 16 precursor T-cell lymphoblastic lymphomas, 10 peripheral T-cell, 4 nasal NK cell, 3 cutaneous anaplastic large cell lymphomas, 1 systemic anaplastic large cell lymphoma and 1 hepatosplenic gamma-delta T-cell lymphoma. Radiotherapy (RT) was performed in 18 patients $(22 \%)$ for the management of NHL prior to ASCT. For 11 patients with a localized aggressive disease, the RT was a planned treatment following 3 cycles of systemic chemotherapy. Palliative RT was delivered to 7 patients due to critical bone involvement, airway compression or various loco-regional problems. The disease stati at mobilization are also shown in Table 1. Twenty-one of the patients $(26 \%)$ that underwent transplantation had a complete response status. Fifty-nine patients had a remaining disease; $19(24 \%)$ an initial partial response, $25(31 \%)$ a chemotherapy-sensitive relapse and 15 (19\%) had either primary refractoriness or a refractory relapse. The IPI at diagnosis and at PBPC mobilization are shown in Table 2. Each factor of the IPI showed a similar distribution at diagnosis and at mobilization, with the exception of the stage, in which stage I/II increased from 35 to $54 \%$ between diagnosis and mobilization. At PBPC mobilization, 70 patients were younger than or equal to 60 years of age. The serum LDH level was elevated in $68 \%$ of patients. The ECOG performance scores were $0 \sim 1$ in 91 and $46 \%$ of patients with stage III and IV diseases, respectively. The number of involved extranodal sites was $0 \sim 1$ in $83 \%$ of patients. When the category of extranodal involvement was modified, 49 patients $(61 \%)$ showed no extranodal involvement and 31 patients (39\%) had at least 1 site of extranodal involvement, which was the reverse of that found at the time of diagnosis (absent 38\%, present 62\%). According to the AAIPI at the time of PBPC mobilization, 20 patients were in the low risk, 28 in the low-intermediate, 25 in the high-intermediate and 7 in the high risk groups.

\section{2) Engraftment and transplant related toxicity}

The median times to neutrophil engraftment $\left(\geq 500 / \mathrm{mm}^{3}\right)$ and platelet transfusion independence $\left(\geq 20,000 / \mathrm{mm}^{3}\right)$ were 11 days, ranging from 8 to 21 , and 14 days, ranging from 9 to
Table 1. Patient characteristics I: Demography at autologous stem cell mobilization

\begin{tabular}{|c|c|c|}
\hline Characteristic & No. & $\%$ \\
\hline \multicolumn{3}{|l|}{ Age, year } \\
\hline Median & 39 & \\
\hline Range & $16 \sim 65$ & \\
\hline \multicolumn{3}{|l|}{ Sex } \\
\hline Male & 51 & 64 \\
\hline Female & 29 & 36 \\
\hline \multicolumn{3}{|l|}{ Histology } \\
\hline$B$ cell & 45 & 56 \\
\hline Diffuse large B cell & 32 & 40 \\
\hline Burkitt & 7 & 9 \\
\hline Mantle cell & 3 & 4 \\
\hline Follicular & 2 & 3 \\
\hline Nodal marginal zone B cell & 1 & 1 \\
\hline$T$ cell & 35 & 44 \\
\hline Precursor $\mathrm{T}$ cell lymphoblastic & 16 & 20 \\
\hline Peripheral $\mathrm{T}$ cell, not otherwise specified & 10 & 13 \\
\hline Nasal NK cell & 4 & 5 \\
\hline Anaplastic large cell, cutaneous & 3 & 4 \\
\hline Anaplastic large cell, systemic & 1 & 1 \\
\hline Hepatosplenic gamma-delta T-cell & 1 & 1 \\
\hline \multicolumn{3}{|l|}{ Radiotherapy prior to mobilization } \\
\hline Not performed & 62 & 78 \\
\hline Performed & 18 & 22 \\
\hline \multicolumn{3}{|l|}{ Disease status at mobilization } \\
\hline Complete response & 21 & 26 \\
\hline Partial response & 19 & 24 \\
\hline Sensitive relapse & 25 & 31 \\
\hline Refractory & 15 & 19 \\
\hline
\end{tabular}


Table 2. Patient characteristics II: International Prognostic Index at diagnosis and autologous stem cell mobilization

\begin{tabular}{|c|c|c|}
\hline \multirow{2}{*}{ Characteristic } & At diagnosis & At mobilization \\
\hline & No. $(\%)$ & No. $(\%)$ \\
\hline \multicolumn{3}{|l|}{ Age (years) } \\
\hline$\leq 60$ & $71(89)$ & $70(88)$ \\
\hline$>60$ & $9(11)$ & $10(13)$ \\
\hline \multicolumn{3}{|l|}{$\mathrm{LDH}^{*}$} \\
\hline Normal & $17(21)$ & $26(33)$ \\
\hline Above normal & $58(73)$ & $54(68)$ \\
\hline \multicolumn{3}{|c|}{ Performance status (ECOG) } \\
\hline $0 \sim 1$ & $64(80)$ & $73(91)$ \\
\hline $2 \sim 4$ & $16(20)$ & 7 (9) \\
\hline \multicolumn{3}{|l|}{ Stage } \\
\hline I, II & $28(35)$ & $43(54)$ \\
\hline III, IV & $52(65)$ & $37(46)$ \\
\hline \multicolumn{3}{|l|}{ Extranodal sites } \\
\hline $0 \sim 1$ & $60(75)$ & $66(83)$ \\
\hline $1<$ & $20(25)$ & $14(18)$ \\
\hline \multicolumn{3}{|c|}{ Modified extranodal involvement } \\
\hline Absent & $30(38)$ & $49(61)$ \\
\hline Present & $50(62)$ & $31(39)$ \\
\hline \multicolumn{3}{|l|}{$\mathrm{IPI}^{\dagger}$} \\
\hline Low & $29(36)$ & $46(58)$ \\
\hline Low intermediate & $21(26)$ & $18(23)$ \\
\hline High intermediate & $18(23)$ & $9(11)$ \\
\hline High & $8(11)$ & $7(9)$ \\
\hline \multicolumn{3}{|l|}{$\mathrm{AAIPI}^{\ddagger}$} \\
\hline Low & $7(9)$ & $20(25)$ \\
\hline Low intermediate & $21(26)$ & $28(35)$ \\
\hline High intermediate & $27(34)$ & $25(31)$ \\
\hline High & 15 (19) & 7 (9) \\
\hline
\end{tabular}

*lactate dehydrogenase, ${ }^{\dagger}$ international prognostic index, ${ }^{*}$ age adjusted international prognostic index.

25. The median in patient stay after stem cell infusion was 21 days, ranging from 14 to 45 .

All patients became transfusion dependent and 65 (81\%) developed an episode of febrile neutropenia requiring intravenous antibiotics. A microbiologically documented infection was noted in 10 patients $(12.5 \%)$. The most common grade 3 or 4 non hematological toxicities were stomatitis and diarrhea, which occurred in $41(51.3 \%)$ and 33 patients (41.3\%), respectively. No other grade 3 or 4 non hematological toxicities were noted. Two patients died of infection 31 and 77 days after ASCT, making the transplant related mortality $2.5 \%$. No cases of secondary malignancy were found.

\section{3) Outcome following ASCT}

The median follow up of surviving patients was 2 years,
Table 3. Univariate analysis of prognostic factors for progression free survival

\begin{tabular}{|c|c|c|}
\hline Variables & $\mathrm{FS}^{\S}$ at 2 years & $\mathrm{p}$ value \\
\hline Cell type & & .3956 \\
\hline B cell & $37 \%$ & \\
\hline $\mathrm{T}$ cell & $30 \%$ & \\
\hline $\mathrm{IPI}^{*}$ at diagnosis & & .1727 \\
\hline Low & $46 \%$ & \\
\hline Low intermediate & $31 \%$ & \\
\hline High intermediate & $18 \%$ & \\
\hline High & $48 \%$ & \\
\hline $\mathrm{AAIPI}^{\dagger}$ at diagnosis & & .3509 \\
\hline Low & $50 \%$ & \\
\hline Low intermediate & $42 \%$ & \\
\hline High intermediate & $32 \%$ & \\
\hline High & $21 \%$ & \\
\hline Age at mobilization & & .645 \\
\hline$\leqq 60$ & $35 \%$ & \\
\hline$>60$ & $33 \%$ & \\
\hline $\mathrm{LDH}^{\ddagger}$ at mobilization & & .1102 \\
\hline Normal & $35 \%$ & \\
\hline$>$ Normal & $33 \%$ & \\
\hline Performance status at mobilization & & .0013 \\
\hline $0 \sim 1$ & $36 \%$ & \\
\hline $2 \sim 4$ & $0 \%$ & \\
\hline Stage at mobilization & & .0033 \\
\hline I or II & $49 \%$ & \\
\hline III or IV & $16 \%$ & \\
\hline Extranodal involvement at mobilization & & .1519 \\
\hline $0 \sim 1$ & $36 \%$ & \\
\hline$>1$ & $36 \%$ & \\
\hline $\begin{array}{l}\text { Modified extranodal involvement } \\
\text { at mobilization }\end{array}$ & & .0049 \\
\hline Absent & $43 \%$ & \\
\hline Present & $17 \%$ & \\
\hline $\mathrm{IPI}^{*}$ at mobilization & & .0013 \\
\hline Low & $44 \%$ & \\
\hline Low intermediate & $24 \%$ & \\
\hline High intermediate & $13 \%$ & \\
\hline High & $0 \%$ & \\
\hline
\end{tabular}

*international prognostic index, ${ }^{\dagger}$ age adjusted international prognostic index, ${ }^{\ddagger}$ lactate dehydrogenase; ${ }^{\S}$ progression free survival.

ranging from 8 to 115 months. The Kaplan Meier estimate of the proportion of patients remaining progression free at 2 years following ASCT was 34\%, with an estimated OS at 2 years of $31 \%$ (Fig. 1A, B). There was a significant different in the estimated 2 year PFS between the patient groups according to the disease status at mobilization; $63 \%$ for complete response, $38 \%$ for partial response, $18 \%$ for chemotherapy sensitive 

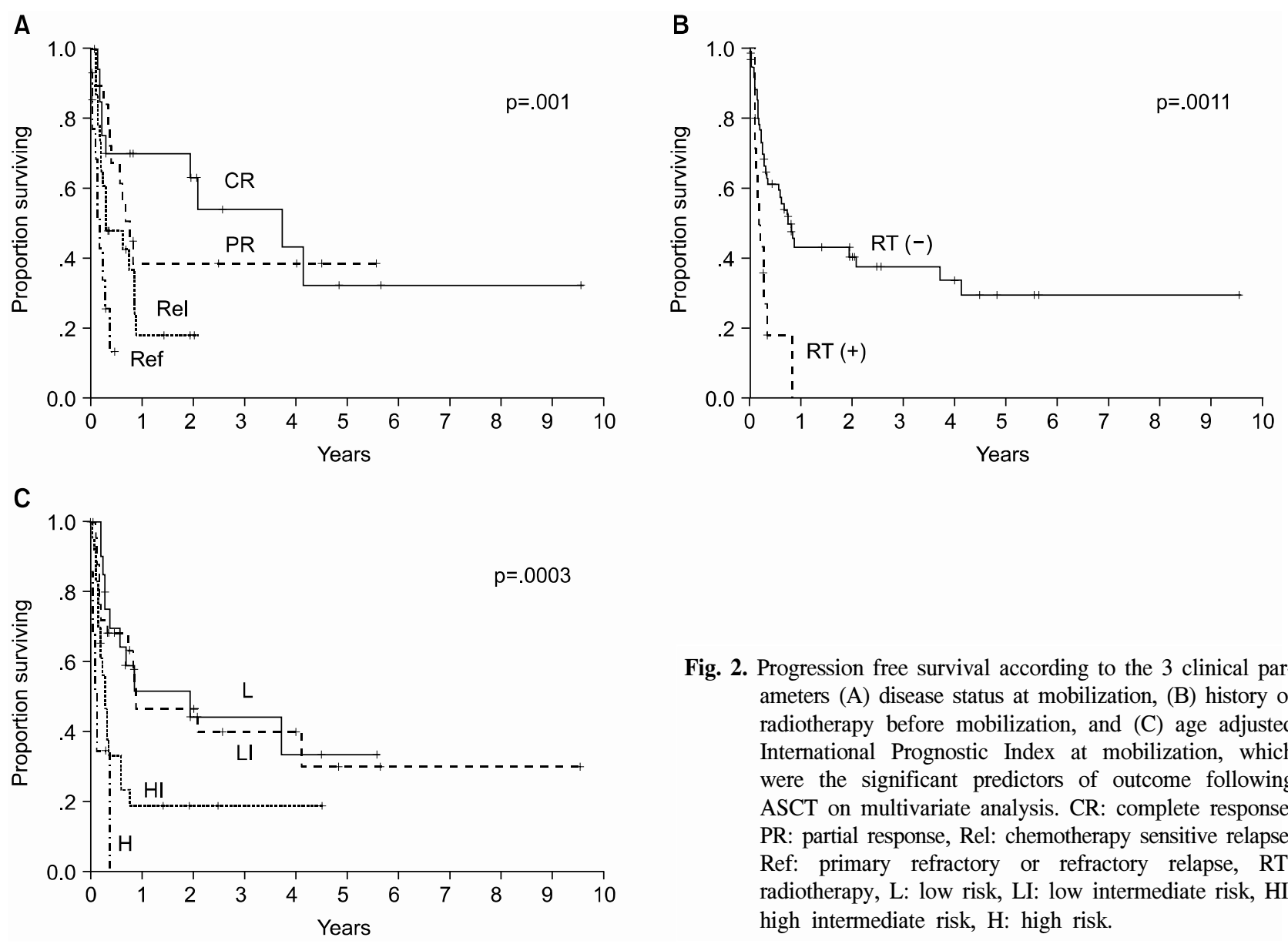

Table 4. Multivariate analysis for progression free survival

\begin{tabular}{lcrc}
\hline Prognostic factors & $\begin{array}{c}\mathrm{p} \\
\text { value }\end{array}$ & $\begin{array}{c}\text { Relative } \\
\text { risk }\end{array}$ & $\begin{array}{c}95 \% \\
\mathrm{CI}^{*}\end{array}$ \\
\hline $\begin{array}{l}\text { Chemotherapy sensitive vs. } \\
\text {-refractory at mobilization }\end{array}$ & .039 & 2.790 & $1.056 \sim 7.376$ \\
$\begin{array}{l}\text { Radiotherapy done vs. } \\
\text { not done }\end{array}$ & .005 & 3.086 & $1.413 \sim 6.739$ \\
$\begin{array}{l}\text { Extranodal involved vs. } \\
\text { not involved at mobilization }\end{array}$ & .450 & .728 & $.319 \sim 1.659$ \\
IPI $^{\dagger}$ at mobilization $\mathrm{L}^{\ddagger}$ or $\mathrm{LI}^{\S}$ & .963 & 1.025 & $.367 \sim 2.857$ \\
$\quad$ vs. HI & & & \\
$\mathrm{AAIPI}^{* *}$ at mobilization $\mathrm{L}^{\ddagger}$ or & .015 & 2.603 & $1.202 \sim 5.635$ \\
$\mathrm{LI}^{\S}$ vs. HI & & & \\
\hline
\end{tabular}

refractory diseases, respectively. A history of radiation treatment for NHL prior to ASCT was also a significant predictor for PFS (Fig. 2B). The PFS at 2 years were 41 and $0 \%$ in patients with no need for radiation treatment before ASCT and for those with a history of prior RT for NHL, respectively $(p=.0011)$. No statistically significant difference was shown in the PFS according to cell type (Table 3 ). The two year PFS were 37 and $30 \%$ for B cell and T cell NHL, respectively $(p=.3956)$. The IPI and AAIPI at diagnosis in our study population were not significant predictors of the outcome following ASCT $(p=.1727$ and $p=.3509$, respectively) (Table 3 ).

\section{4) mAAIPI as predictor of outcome}

When the components of the mIPI were assessed in the univariate analysis using the log rank test (Table 3), the performance status and stage at PBPC mobilization were significant predictors of PFS. Age $(p=.645)$, LDH $(p=.1102)$ and extranodal involvement $(p=.1519)$ were not predictive of PFS. When extranodal involvement was newly categorized as either absent or present, those with no extranodal site at mobilization showed 2 year PFS of $43 \%$ in comparison to $17 \%$ for those with extranodal involvement $(p=.0049)$. The PFS at 2 years were significantly different among patients grouped by the mIPI (Table 3): 44, 24, 13 and $0 \%$ for the low, low intermediate, high intermediate and high risk groups, respectively $(\mathrm{p}=.0013)$. The survival of all patients was assessed to see the predict- 
ability of the mAAIPI on the PFS. The PFS at 2 years were as follows: 44, 46, 19 and $0 \%$ for the low, low intermediate, high intermediate and high risk groups, respectively (Fig. 2C). The mAAIPI was able to predict the PFS $(p=.0003)$. A multivariate analysis for factors affecting the PFS, using a Cox proportional hazards model, demonstrated that disease status at mobilization, prior RT for NHL and mAAIPI as significant and independent predictors of survival (Table 4).

\section{DISCUSSION}

The International Consensus Conference on ASCT in aggressive NHL reported that patients with a chemosensitive relapse, those with a primary refractory status and some complete responders are suitable candidates for high dose therapy (20). The jury discussed the role of ASCT in slow or incomplete responders to initial induction therapy, and did not exclude the possibility of a beneficial effect. The report commented that the benefit of ASCT in partial responders remains to be defined. Those showing a chemorefractory relapse were assessed as inappropriate for ASCT; the quality of the evidence; however, was not so affirmative. In the current study, ASCT was performed for those with a complete response with a high risk of relapse at initial presentation, a partial response to initial induction chemotherapy, a chemosensitive relapse and a primary refractory or refractory relapse. Refractory patients showed a good performance, and had no other treatment modality, with the exception of ASCT for the control of their disease. On the basis of the report from the International Consensus Conference on ASCT in aggressive NHL (20), the patients in our study were appropriate candidates for ASCT.

It is inevitable that the disease status at the time of ASCT is a prognostic indicator. Reports on Korean NHL patients showed that those with a chemotherapy sensitive disease had better survival than those with a resistant disease. Also, it would be better if the prognostic factors within each strata of the disease status at mobilization of PBPC could be analyzed. Because the current report is based on the experience from of a single institution, which resulted in a small number of study patients, all NHL patients treated by ASCT at the Asan Medical Center were included. Attempts were also made to find every possible predictive indicator for the outcome of ASCT using a multivariate analysis. The small number of patient, with the inclusion of different disease stati, could also be either positive or negative aspects of the current study. If clinical factors for predicting the outcome following ASCT could be found within this small and inhomogeneous population, these would be very influential. The univariate analysis showed that the IPI at diagnosis was not predictive of the outcome following ASCT, which seems plausible. The closer the assessment of the clinical parameters to ASCT, the more predictive these factors might be of the outcome. Here, the clinical situation at PBPC mobilization was used. Because the patients' disease status at the time of PBPC mobilization was evaluated to make the flow of clerical work smooth, the time point was actual and practical for the current study.

Attempts were also made to try and modify the criteria of extranodal involvement in this study. The frequency of extranodal involvement is higher in Korea than in Western countries (21). In our other unpublished analysis of NHL patients at the Asan Medical Center, the statistical significance of extranodal involvement was greater when the presence or absence, rather than $0 \sim 1$ or $>1$ site, as in the original IPI system, was used. Our present study also showed the significance of extranodal involvement when the category was modified from that used in the IPI system. This implies that it might be necessary to investigate which categorization of the extranodal involvement would be optimal for those with a high frequency of extranodal involvement.

In the multivariate analysis of those factors statistically significant by the univariate analysis, the performance status and stage at PBPC were not included, as these are components of the mIPI or mAAIPI. A modified categorization of the extranodal involvement was applied to the multivariate analysis, as this was relatively different from that of the original IPI category. The Cox proportional hazards model conducted in the current study revealed that modified extranodal involvement and mIPI were not independent predictors of the outcome following ASCT in NHL patients. Also, it might be possible to discover if the disease status at PBPC mobilization, history of prior RT and mAAIPI are independent prognostic indicators following ASCT in current NHL patients. In the multivariate analysis, the mIPI lost its significance, as evidenced in the univariate data analysis. $88 \%$ of the patients were aged 60 years or younger at mobilization, which might make the significance of the age category less meaningful. As described earlier, the original categorization of the extranodal involvement might be less meaningful for patients with a higher frequency of extranodal manifestations. This might also have contributed to the loss of significance for the mIPI in the multivariate analysis. Despite modification, the new extranodal involvement category was not shown to have a role as a single independent prognostic indicator in the Cox proportional hazards model. Because a previous history of RT was shown to have such a strong statistical power in the current study, but with no reasonable explanation, and the disease status being a well known factor that also showed its definite significance in this analysis. And as the mAAIPI is a combination of significant prognostic factors, which was shown to have a unique role as an indicator of prognosis in the Cox regression model, the extranodal manifestation itself, even when modified, might have lost its role as an independent prognostic factor. This modified categorization for the extranodal involvement might have significance when combined with other IPI categories, such as the LDH level, performance status or stage. The small sample size of our study made this analysis difficult.

It is interesting that RT itself was a significant indicator of the prognosis, even after the Cox regression analysis. Eighteen patients had been exposed to RT prior to PBPC mobilization, and had many adverse factors. Fourteen of these had a refractory disease status, 9 extranodal involvements and 6 had a high or high intermediate mAAIPI. Although patients with a prior history of RT tended to have extra adverse factors, the multivariate analysis showed that a previous history of RT had unique significance as a prognostic factor. The mAAIPI also retained its significance in the multivariate analysis. Recently, AAIPI at transplantation was reported to have prognostic 
significance in diffuse large B cell lymphomas undergoing ASCT (13). Even though a time lag between PBPC mobilization and the ASCT procedure might exist, the clinical change or difference should be negligible. Because the actual decision, restaging and request of medical insurance coverage are performed just prior to PBPC mobilization in our center, using the time point of mobilization rather than transplantation would seem more reasonable and practical.

The OS and PFS of all the patients were poor. The survival of each disease status also showed inferior results to those of previously reported (18). This poor result was assumed might have been caused due to intrinsic factors making the prognosis poorer. The intrinsic adverse prognostic factors in the patients of the current study were the greater number of NHL patients with the $\mathrm{T}$ cell phenotype, more prevalent extranodal manifestations, and so on, which are general characteristics of Korean NHL patients. To improve the survival of Korean NHL patients following ASCT, the predictors of outcome revealed in this current study should be employed.

\section{CONCLUSIONS}

In Korean NHL patients, with inherent adverse prognostic factors, the disease status at PBPC mobilization, prior RT history and mAAIPI were found to be independent predictors of the outcome following ASCT. This powerful prognostic tool, the mAAIPI, should be applied to evaluate potential suitable candidates of ASCT and to compare the results of forthcoming approaches in ASCT.

\section{ACKNOWLEDGMENT}

We thank the house staff members and nurses at 84 ward of the Asan Medical Center for their dedication and excellent patient care.

\section{REFERENCES}

1. Mink SA, Armitage JO. High dose therapy in lymphomas: a review of the current status of allogeneic and autologous stem cell transplantation in Hodgkin's disease and non Hodgkin's lymphoma. Oncologist. 2001;6:247-56.

2. Appelbaum FR, Herzig GP, Ziegler JL, Graw RG, Levine AS, Deisseroth AB. Successful engraftment of cryopreserved autologous bone marrow in patients with malignant lymphoma. Blood. 1978;52:85-95.

3. Philip T, Armitage JO, Spitzer G, Chauvin F, Jagannath S, Cahn JY, et al. High dose therapy and autologous bone marrow transplantation after failure of conventional chemotherapy in adults with intermediate grade or high grade non Hodgkin's lymphoma. N Engl J Med. 1987;316:1493-8.

4. Vose JM, Anderson JR, Kessinger A, Bierman PJ, Coccia P, Reed EC, et al. High dose chemotherapy and autologous hematopoietic stem cell transplantation for aggressive non Hodgkin's lymphoma. J Clin Oncol. 1993;11:1846-51.

5. Petersen FB, Appelbaum FR, Hill R, Fisher LD, Bigelow CL, Sanders JE, et al. Autologous marrow transplantation for malignant lymphoma: a report of 101 cases from Seattle. J Clin Oncol. 1990;8:638-47.

6. Rapoport AP, Lifton R, Constine LS, Duerst RE, Abboud CN, Liesveld JL, et al. Autotransplantation for relapsed or refractory non Hodgkin's lymphoma (NHL): long term follow up and analysis of prognostic factors. Bone Marrow Transplant. 1997; 19:883-90.

7. Phillips GL, Fay JW, Herzig RH, Lazarus HM, Wolff SN, Lin HS, et al. The treatment of progressive non Hodgkin's lymphoma with intensive chemoradiotherapy and autologous marrow transplantation. Blood. 1990;75:831-8.

8. A predictive model for aggressive non Hodgkin's lymphoma. The International Non Hodgkin's Lymphoma Prognostic Factors Project. N Engl J Med. 1993;329:987-94.

9. Hermans J, Krol AD, van Groningen K, Kluin PM, Kluin Nelemans JC, Kramer $\mathrm{MH}$, et al. International prognostic index for aggressive non hodgkin's lymphoma is valid for all malignancy grades. Blood. 1995;86:1460-3.

10. Blay J, Gomez F, Sebban C, Bachelot T, Biron P, Guglielmi $\mathrm{C}$, et al. The international prognostic index correlates to survival in patients with aggressive lymphoma in relapse: analysis of the PARMA trial. Blood 1998;92:3562-8.

11. Moskowitz CH, Nimer SD, Glassman JR, Portlock CS, Yahalom J, Straus DJ, et al. The international prognostic index predicts for outcome following autologous stem cell transplantation in patients with relapsed and primary refractory intermediate grade lymphoma. Bone Marrow Transplant. 1999;23: 561-7.

12. Cuttica A, Zallio F, Ladetto M, Di Nicola M, Caracciolo D, Magni M, et al. Patients with high risk aggressive lymphoma treated with frontline intensive chemotherapy and autografting evidence of marked differences in outcome between patients with age adjusted International Prognostic Index scores 2 and 3. Cancer. 2003;98:983-92.

13. Hamlin PA, Zelenetz AD, Kewalramani T, Qin J, Satagopan $\mathrm{JM}$, Verbel D, et al. Age adjusted international prognostic index predicts autologous stem cell transplantation outcome for patients with relapsed or primary refractory diffuse large B cell lymphoma. Blood. 2003;102:1989-96.

14. National Cancer Institute sponsored study of classifications of non Hodgkin's lymphomas: summary and description of a working formulation for clinical usage. The Non Hodgkin's Lymphoma Pathologic Classification Project. Cancer. 1982;49: 2112-35.

15. Harris NL, Jaffe ES, Diebold J, Flandrin G, Muller Hermelink $\mathrm{HK}$, Vardiman J, et al. World health organization classification of neoplastic diseases of the hematopoietic and lymphoid tissues: report of the clinical advisory committee meeting: Airlie House, Virginia, November 1997. J Clin Oncol. 1999; 17:3835-49.

16. Cheson BD, Horning SJ, Coiffier B, Shipp MA, Fisher RI, Connors JM, et al. Report of an international workshop to standardize response criteria for non Hodgkin's lymphomas. J Clin Oncol. 1999;17:1244-53.

17. Suh C, Kim HJ, Kim SH, Kim S, Lee SJ, Lee YS, et al. Low dose lenograstim to enhance engraftment after autologous stem cell transplantation: a prospective randomized evaluation of two different fixed doses. Transfusion. 2004;44:533-8.

18. Jantunen E, Kuittinen T, Nousiainen T. BEAC or BEAM for high dose therapy in patients with non Hodgkin's lymphoma? A single centre analysis on toxicity and efficacy. Leuk Lymphoma. 2003;44:1151-8.

19. Kim H, Sohn HJ, Kim SE, Kang HJ, Park S, Kim S, et al. Lymphocyte recovery as a positive predictor of prolonged survival after autologous peripheral blood stem cell transplantation in $\mathrm{T}$ cell non Hodgkin's lymphoma. Bone Marrow 
Transplant. 2004;34:43-9.

20. Shipp MA, Abeloff MD, Antman KH, Carroll G, Hagenbeek $\mathrm{A}$, Loeffler $\mathrm{M}$, et al. International consensus conference on high dose therapy with hematopoietic stem cell transplantation in aggressive non Hodgkin's lymphomas: report of the jury.
J Clin Oncol. 1999;17:423-9.

21. Kim HT, Im YH, Suh CI, Park YS, Kang WK, Heo DS, et al. Malignant lymphomas in Korea. J Korean Cancer Assoc. 1992;24:92-101. 